Original Research Paper

\title{
Total Phenolic, Resveratrol Content, Antioxidant and Antimicrobial Activity in Five Cultivars of Peanut Sprouts
}

\author{
${ }^{1,2}$ Qing Liu, ${ }^{1,2}$ Shaoqi Li, ${ }^{1,2}$ Yao Zhang, \\ ${ }^{1,2}$ Huaiyuan Zhang, ${ }^{1,2}$ Haifang Xiao, ${ }^{3}$ Tian Tian and ${ }^{1,2}$ Yuanda Song \\ ${ }^{1}$ Colin Ratledge Center for Microbial Lipids, School of Agricultural Engineering and Food Science, \\ Shandong University of Technology, 266 Xincun West Road, Zibo 255000, People's Republic of China \\ ${ }^{2}$ Key Laboratory of Shandong Provincial, Universities for Technologies in Functional Agricultural Products, \\ Shandong University of Technology, 266 Xincun West Road, Zibo 255000, People's Republic of China \\ ${ }^{3}$ School of Traditional Chinese Medicine, Beijing University of Chinese Medicine, No. 11, \\ Bei San Huan Dong Lu, Chaoyang District, Beijing 100029, People's Republic of China
}

\begin{abstract}
Article history
Received: 03-12-2019

Revised: 14-01-2020

Accepted: 08-02-2020

Corresponding Author:

Yuanda Song

Colin Ratledge Center for

Microbial Lipids, School of

Agricultural Engineering and

Food Science, Shandong

University of Technology, 266

Xincun West Road, Zibo

255000, People's Republic of

China

Email: ysong@sdut.edu.cn
\end{abstract}

\begin{abstract}
Peanut sprout is one of the potential natural sources of bioactive substances. In this study, five different peanut cultivars were conducted to germination, the change in total phenolic compounds, resveratrol content, antioxidant and antimicrobial activity were investigated. The sprouts of five peanut cultivars (Fuhua, Silihong, Dabaisha, Xiaobaisha and Huayu) were extracted using $75 \%$ ethanol for $3 \mathrm{~h}$ and the crude extracts were collected. When the five peanut kernels were germinated at $25^{\circ} \mathrm{C}$ for 5 days, the total phenolic compounds increased significantly from range of 0.55 to $0.96(\mu \mathrm{g}$ Gallic acid/g) up to the range of 1.97 to 2.44 ( $\mu$ g Gallic acid/g) and the resveratrol contents showed similar trend, from range of 8.94 to $15.62 \mu \mathrm{g} / \mathrm{g}$ up to the range of 31.74 to $46.49 \mu \mathrm{g} / \mathrm{g}$ depending upon peanut cultivar. Among the five cultivars, Xiaobaisha showed the highest phenolic content [2.44 ( $\mu \mathrm{g} \mathrm{Gallic} \mathrm{acid/g)],} \mathrm{expressed} \mathrm{the} \mathrm{highest} \mathrm{2,2-diphenyl-1-picrylhydrazyl}$ scavenging value $[(84.86 \pm 4.02) \mathrm{mmol} / \mathrm{L} \mathrm{TE} / \mathrm{g}$ dry weight $]$ and ferric ion reducing antioxidant power scavenging value $[(92.98 \pm 3.71) \mathrm{mmol} / \mathrm{L} \mathrm{TE} / \mathrm{g}$ dry weight] on the fifth day. In the antimicrobial evaluation the five sprout extracts were highly effective in inhibitory zone assay followed by Minimum Inhibitory Concentration (MIC) and Minimum Bactericidal Concentration (MBC) analysis against five pathogen microbial. In comparison with other peanut cultivars, the inhibitory effects were strongest in Silihong, the average MIC and MBC values against 5 spoilage bacteria were 10.66 and $28.52 \mathrm{mg} / \mathrm{mL}$, respectively. In summary, this work provides evidences that Xiaobaisha and Silihong might be applied as antioxidant and antibacterial agent in food and pharmaceutical industries.
\end{abstract}

Keywords: Peanut Sprout, Resveratrol, Phenolic Compound, Antioxidant Activity, Antimicrobial Activity

\section{Introduction}

Under oxidative stress condition, free radicals that generated via metabolic process plays a key role in the onset of various pathophysiological disorders including cancer, proliferative diseases, diabetes mellitus and early aging (Manoharan et al., 2019). Numerous synthetic drugs have been used to inhibit the interaction of free radicals and pathogenic microbes which could worsen the severity of a disease. Unfortunately, selective resistance of bacteria toward traditional and synthetic drugs have emerged as a new challenge. As the synthetic drugs are non-specific and carries side effects, the demand for natural alternatives by consumers are increasing (Verraes et al., 2013; EFSA, 2017). Thus, many studies are being carried out to look for natural alternative for antioxidant and antimicrobial agents.

Peanut (Arachis hypogaea) is an annual herbaceous plant which belongs to the Fabaceae family. In China, peanuts are common food and one of the most important oilseeds and the annual production of peanuts exceeds 14 million tons (Zhang, 2016). In recent years, peanut 
sprout has been attracted extensive interests of the public due to its benefits to human health. The sprouts are rich in phytochemicals, proteins, vitamins and minerals (Kim et al., 2011). Many studies have revealed that varieties of phenolic substances such as resveratrol, arachidin-1 and piceatannol have been found in peanut sprout (Ahn et al., 2012; Santos et al., 2013; Xiong et al., 2014). Reports found that the phenolic compounds in peanut sprouts, especially the resveratrol, have the capacity of anti-aging (Park et al., 2012), anticancer (Whitlock and Baek, 2012), anti-inflammatory (Ghanim et al., 2011) and the prevention of cardiovascular disease (Nakata and Inoue, 2014). And it was indicated that the resveratrol biosynthesis was enhanced by subjecting peanut to germination (Wang et al., 2005).

With the backdrop information the present study was intended and carried out to determine the total resveratrol and phenolic compound, antioxidant and antimicrobial activity of the spouts extracts of five cultivars (Fuhua, Silihong, Dabaisha, Xiaobaisha and Huayu) growing in China. The resveratrol content, antioxidant and antimicrobial activity studies of these five germinated peanuts by far have not been reported yet. The five cultivars were subjected to germination for 5 days and the ethanol extraction was conducted. The total resveratrol and phenolic content were then investigated and compared each other among different cultivars and germination time. The relationship of total phenolic compounds and antioxidant and antimicrobial assay was also studied.

\section{Materials and Methods}

\section{Materials}

The five sound and mature peanut kernels: Fuhua, Silihong, Dabaisha, Xiaobaisha and Huayu used in this study were kindly provided by Professor Tian Tian (Beijing University of Chinese Medicine, China).

\section{Chemicals, Reagents and Baterial Strains}

Standard compounds used for content determination and antioxidant evaluation including resveratrol, ascorbic acid, gallic acid, 2,2-Diphenyl-1-Picrylhydrazyl (DPPH), 3ethylbenzothiazoline-6-sulfonic acid (ABTS) and FolinCiocalteu reagent were purchased from Sigma-Aldrich. 6Hydroxy-2,5,7,8-tet-ramethylchroman-2-carboxylic acid (Trolox) was purchased from Fluka (Buchs, Germany). HPLC grade solvents including ethanol, methanol, acetonitrile and formic acid were purchased from Tedia Company Inc. (OH, USA). Other reagents an solvents with analytical grade such as Pyrogallol, ferrous oxide and salicylic acid were purchased from Sinopharm Chemical Reagent Co., Ltd (shanghai, China). The water used in the experiment was produced by a Milli-Q system (MA, USA).

All the bacterial strains including Escherichia coli (CMCC 44102), Bacillus Subtilis (CCTCCAB206681), Salmonella enterica (BNCC132287), Staphylococcus aureus subsp. aureus (CMCC26003) and Pseudomonas aeruginosa (BNCC 125486) were purchased from BeNa Culture Collection (Beijing, China) used for Antimicrobial test in vitro.

\section{Peanut Germination}

For each batch, 100 visibly sound kernels were sorted, weighted and soaked into water overnight at the ambient temperature $\left(24-26^{\circ} \mathrm{C}\right)$. The kernels were placed on a plastic net tray and germinaed under dark in BE-801 growth chamber (Bovoee, China) at $25^{\circ} \mathrm{C}$ and $85 \%$ relative humidity. The kernels were under $10 \mathrm{~min}$ of spray tap water with pauses of 20 min for 5 days. After 3 days of incubation, the ungerminated kernels (without emergence of germ tube) were discarded to eliminate molding. The total germination time was 5 days. For each day, the numbers and weights of five cultivar sprouts were counted and weighed, respectively.

\section{Peanut Sprout Preparation and Extraction}

The germinated peanut sprouts were harvested and weighed and followed by lyophilization (Labconco, USA). After lyophilization and weight determination, the dried materials were grinded into sprout powders and sieved to 60-mesh. The powders were sealed in polyethylene ziplock bags and stored under $-20^{\circ} \mathrm{C}$ for resveratrol and phnolic extraction. Peanut sprout powders were extracted with $75 \%$ ethanol for $3 \mathrm{~h}$. The extract evaporation was carried out using a rotary evaporator (EYELA N1000, Japan) at $45^{\circ} \mathrm{C}$ under a vaccum pressure. The crude extract was collected and transformed into a 50 $\mathrm{mL}$ volumetric flask, thereafter, the volume was adjusted by adding $75 \%$ ethanol to meet the scale line.

\section{Scavenging Ability Measured by the DPPH Radical} Assay

Radical scavenging activity was performed using a method developed by Akharaiyi et al. (2019). $2 \mathrm{~mL}$ of crude extract was added to $2 \mathrm{~mL}$ of $0.2 \mathrm{mmol} / \mathrm{L} \mathrm{DPPH}$ radical. For making standard curve, extra ethanol was added, to provide a total reaction volume of $4 \mathrm{~mL}$. Ethanol was used as a blank and the reaction mixture was vigorously shaken and incubated in the dark for 30 min. The reduction of DPPH radical absorption was measued at optical density of $517 \mathrm{~nm}$ at room temperatue. A calibation curve was prepared by measuring the reduction in absorbance of the DPPH solution in the presence of different concentrations of Trolox. Results were expressed as Trolox equivalents antioxidant capacity (mmol/L TE/g dry weight). All determinations were performed in triplicate.

\section{Scavenging Ability Measured by Stable ABTS Radical Action}

ABTS assays were performed as described by Lee et al. (2016), with some modifications. ABTS was dissolved in 
water to obtain a $7 \mathrm{mM}$ stock solution. ABTS radical cation solution was prepared by diluting ABTS stock solution with $2.4 \mathrm{mM}$ potassium persulfate 1:1 (v/v), followed by incubation for $16 \mathrm{~h}$ at room temperature and in the dark. ABTS redical solution was adjusted with phosphatedbuffered saline ( $\mathrm{pH} 7.4$ ) to an absorbance between $0.75-0.8$ at $734 \mathrm{~nm} .180 \mu \mathrm{L}$ of the ABTS reagent was mixed with 20 $\mu \mathrm{L}$ of each test sample. The absorbance was measured at $734 \mathrm{~nm}$. The scavenging capacity of the extracts was compared with that of trolox. The antioxidant activity was expressed as mmol of $\mathrm{TE} / \mathrm{g}$ of dry weight. All determinations were performed in triplicate.

\section{Ferric Reducing Antioxidant Power Assay (FRAP)}

Reducing power was determined according to the method of Ożarowski et al. (2016) with slight modifications. FRAP reagent was prepared by mixing 10 $\mathrm{mM}$ 2,4,6-tripyidyl-s-triazine (TPTZ) in $40 \mathrm{mM} \mathrm{HCl}$, $20 \mathrm{mM}$ ferric chloride and $300 \mathrm{mM}$ acetate buffer $(\mathrm{pH}$ 3.4) using the ratio $1: 1: 10(\mathrm{v} / \mathrm{v} / \mathrm{v}) .10 \mu \mathrm{L}$ of each test sample solution was well mixed with $300 \mu \mathrm{L}$ FRAP reagent and it was allowed to react for $5 \mathrm{~min}$ in a water bath $\left(37^{\circ} \mathrm{C}\right)$. The absrbance was measured at $593 \mathrm{~nm}$ in three replicate experiments. Trolox was used as a standard and the antioxidant activity was exptressed as mmol of TE/g of day weight.

\section{Total Phenolic Content Determination}

Total phenolic content of the five peanut sprout extracts was conducted using the Folin-Ciocalteu reagent method according to Hameed et al. (2017) with minor modification. The reaction was carried out by mixing $200 \mu \mathrm{L}$ sample extract with $300 \mu \mathrm{L} 10 \%$ (v/v) FolinCiocaleu reagent and then adding $500 \mu \mathrm{L}$ sodium carbonate $(20 \%, \mathrm{w} / \mathrm{v})$ solution. The reaction was incubated at room temperature for $20 \mathrm{~min}$ in the dark and the absorbance was measured at $765 \mathrm{~nm}$. Gallic acid was used as the standard and the concentration of total phenolic was expressed as Gallic Acid Equivalent (GAE) ( $\mu$ g gallic acid/g dry weight). All determinations were carried out in triplicates.

\section{Resveratrol Concentration Determination}

The qualification and quantification of resveratrol was performed using HPLC method described by Zhang et al. (2015), with some modification. The peanut sprout crude extract was filtered through a 0.22 $\mu \mathrm{m}$ microfilter before injection into HPLC. The separation was carried out by Poroshell120 EC-C18 column $(4.6 \times 150 \mathrm{~mm}$ i.d., Agilent, USA). The mobile phase consisted of acetonitrile: water (45: $55, \mathrm{v} / \mathrm{v})$ and was run at a constant flow rate of $1 \mathrm{~mL} / \mathrm{min}$. The ingection volume was $10 \mu \mathrm{L}$ and the column temperature was set at $25^{\circ} \mathrm{C}$. The chromatograms were detected using a DAD detector at $306 \mathrm{~nm}$.

\section{Anti-Microbiological Activity Assay}

Five pathogenic and spoilage organisms'viz. Escherichia coli, Bacillus Subtilis, Salmonella enterica, Staphylococcus aureus subsp. aureus and Pseudomonas aeruginosa were used in assay protocol. The freeze dried cultures were activated and cultures were maintained at refrigetation temperature by sub culturing. The required bacterial population was obtained by serial dilution using sterile peptone water. The dose rate of the inoculums was standardized on the basis of cell number in the inoculums. The dose rate of the above mentioned microbial cultures was optimized in the range of $10^{4}-10^{5} \mathrm{cfu} / \mathrm{mL}$. Pour plate technique was used for inoculation and media solidification. $1 \mathrm{~mL}$ of the test culture $\left(10^{4}-10^{5} \mathrm{cfu} / \mathrm{mL}\right)$ were uniformly distributed by pouring $15-20 \mathrm{~mL}$ of pre-sterilized media and allowed for solidification. In each plate 4 wells $(8 \mathrm{~mm}$ diameter) were made using sterile borer. About $100 \mu \mathrm{L}$ of each extracts were poured into well onto solid media in nutrient agar for all the test organisms and incubated at $37^{\circ} \mathrm{C}$ for $24 \mathrm{~h}$. The diameters of inhibitory zone surounding the wells were measured using digital vernier calipers.

\section{Minimum Inhibitory Concentration (MIC) and Minimum Bactericidal Concentration (MBC) Assay}

Ninety-six well plates (SPL Co. Ltd, Pocheon, Korea) were used. Each $100 \mathrm{~mL}$ of $1: 1$ (v:v) LB broth $(2 \mathrm{x})$ and $2 \%$ antimicrobial stock solution were mixed in 96-well plates to give final concentrations of $1 \%$. Serial 2-fold dilutions were then performed using $100 \mathrm{~mL}$ of LB broth. Next, each well plate was inoculated with 20 $\mu \mathrm{L}$ of the cultured strains of spoilage bacteria and were incubated at $37^{\circ} \mathrm{C}$ for $24 \mathrm{~h}$. Optical densities of the cultures at $595 \mathrm{~nm}$ were measured after $24 \mathrm{~h}$ incubation, following adjustment to 0 by dilution in fresh media. The MIC and MBC values were determined at the lowest concentration based on the turbidity of two subjects at the same concentration at which Optical densities at $595 \mathrm{~nm}$ were less than 0.5 and 0.1, respectively (Ji et al., 2008).

\section{Statistical Analysis}

All the experiments were repeated three times and parameters were analysed in triplicate $(n=9)$. Statistical One-way ANOVA was calculated using SPSS software version 22.0. Differences were considered statistically significant at the $P<0.05$ level.

\section{Results and Discussion}

\section{Total Phenolic Content}

The five peanut cultivars (Fuhua, Silihong, Dabaisha, Xiaobaisha and Huayu) were germinated for 1-5 days and the total phenolic contents were evaluated using the Folin-Ciocalteau method. The results are as shown in Fig. 1, the total phenolic contents of five peanut cultivars 
were mainly increased from one to five days germination. From the result, Fuhua, Silihong, Xiaobaisha and Huayu all showed gradually increasing trends of phenolic content during the five-days germination. Among them, Xiaobaisha showed higher phenolic content than the others at the extended germination time and reached the highest value $(2.44 \pm 0.06 \mu \mathrm{g}$ gallic acid $/ \mathrm{g})$ on the fifth day. In contrast to these four cultivars, a significant decrease in the total phenolic content on the second day of germination $(0.79 \pm 0.05 \mu \mathrm{g}$ gallic acid $/ \mathrm{g})$ was observed for the Dabaisha cultivar. Later, the phenolic content increased to $2.20 \pm 0.04 \mu \mathrm{g}$ gallic acid $/ \mathrm{g}$ on the fifth day of germination.

\section{Resveratrol Content}

Resveratrol is a natural stilbene phytoalexin with benefits to human health. The results in Fig. 2 demonstrated the total resveratrol content of five peanut cultivars measured by the HPLC method. Five cultivars indicated a similar pattern, producing the lowest resveratrol content on the first day of germination and steadily increase in germination time. The highest resveratrol content on the fifth day of germination was $46.49 \pm 1.60 \mu \mathrm{g} / \mathrm{g}, 34.65 \pm 0.48 \mu \mathrm{g} / \mathrm{g}, 31.74 \pm 0.60 \mu \mathrm{g} / \mathrm{g}$, $38.22 \pm 0.96 \mu \mathrm{g} / \mathrm{g}$ and $31.94 \pm 1.28 \mu \mathrm{g} / \mathrm{g}$ for Fuhua, Silihong, Dabaisha, Xiaobaisha and Huayu, respectively.

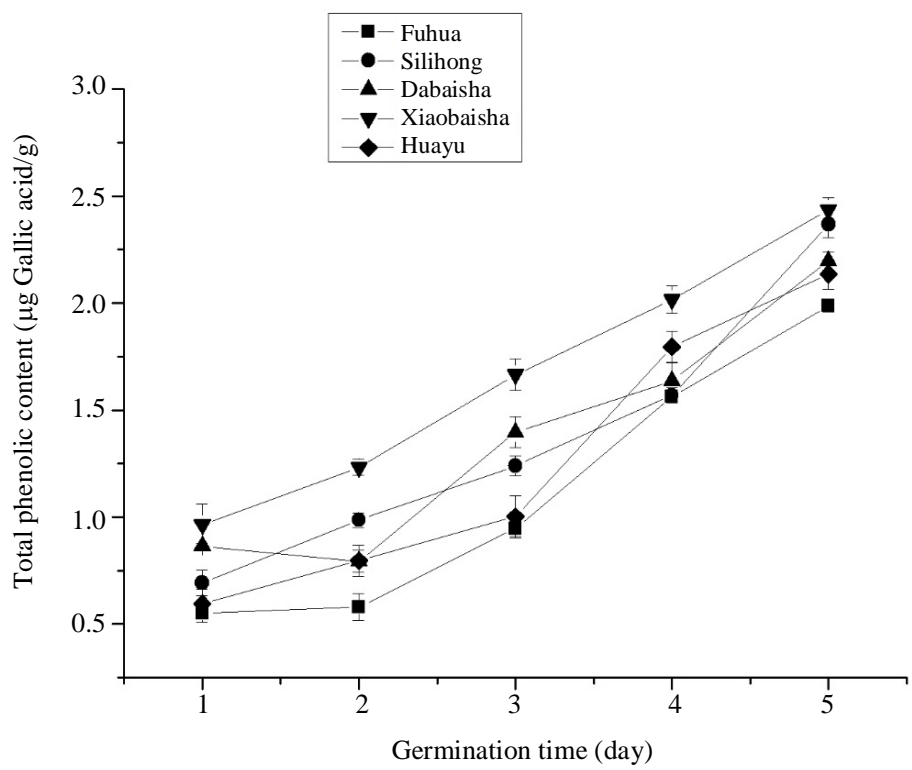

Fig. 1: Total phenolic content of five cultivars of peanut sprout germinated during 1-5 days

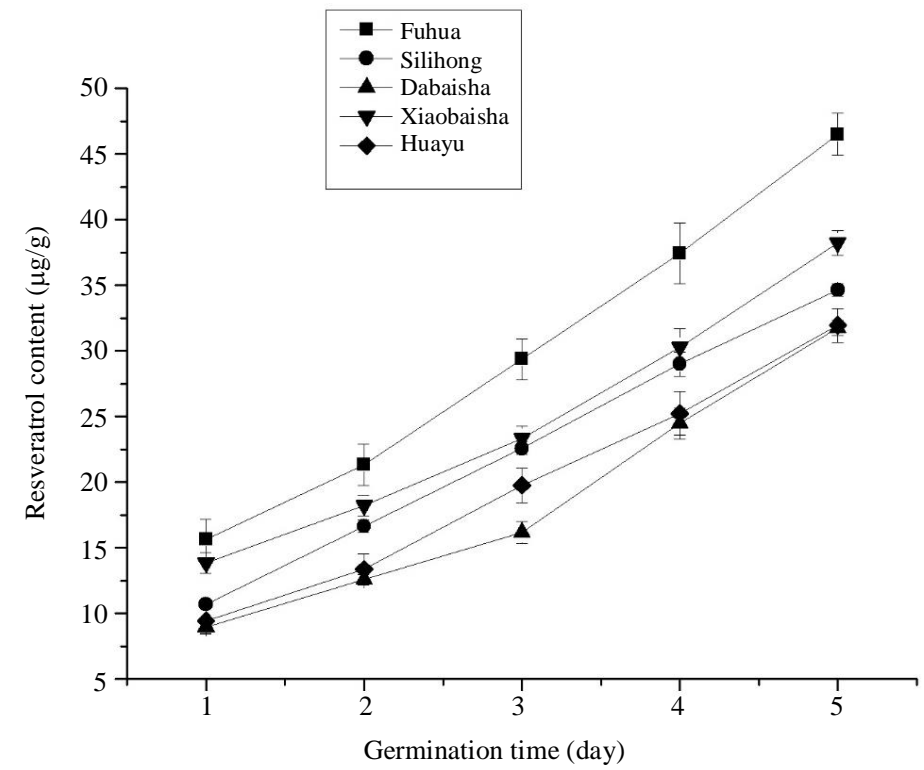

Fig. 2: Total resveratrol content of five cultivars of peanut sprout germinated during 1-5 days 


\section{Antioxidant Activity}

In general, becasuse the antioxidant compounds in plant extracts and chemically diverse and structurally complex, no single assay is suitable to accurately determine the antioxidant activity for all compounds present in a sample (Prior et al., 2005); the use of multple assays is therefore prefrable. In our study, antioxidant activity was determined using three different in vitro methods. The ABTS assay is based on the electron transfer mechanism involving ABTS radicals while for the DPPH assay, an reaction between DPPH radicals and anioxidants achieved through hydrogen atom and electron transfer mechanisms (Huang et al., 2005). On the other hand, the FRAP assay is based on electron trasfer mechanism without the involvement of free radcals (Huang et al., 2005).

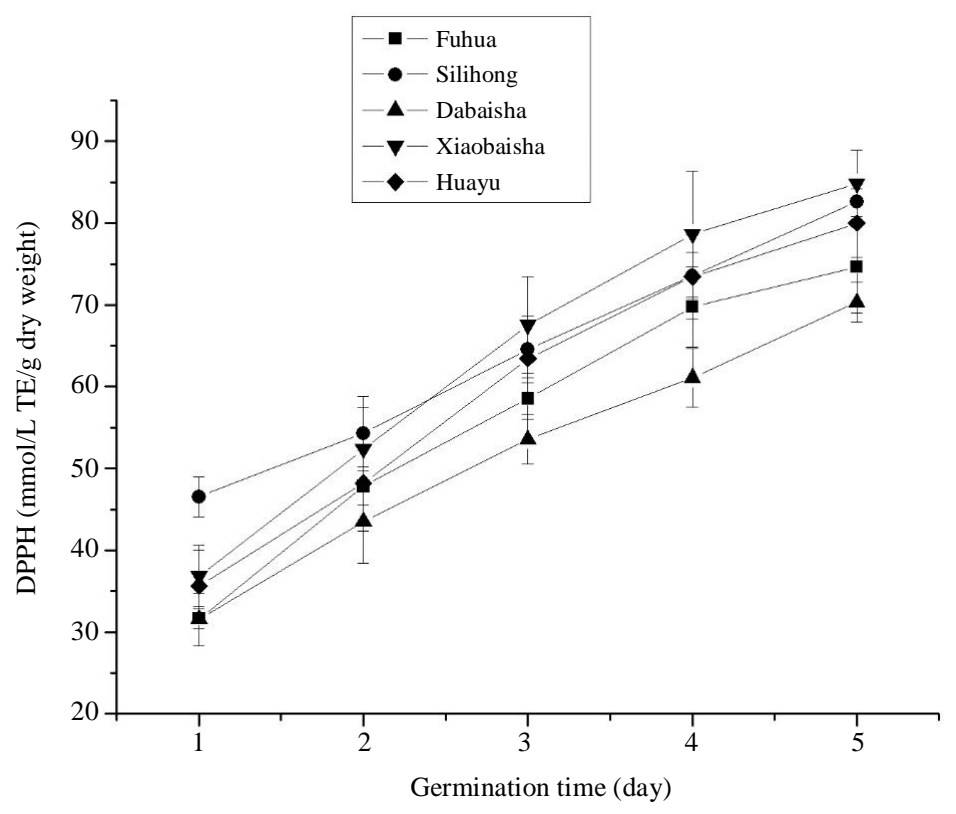

(A)

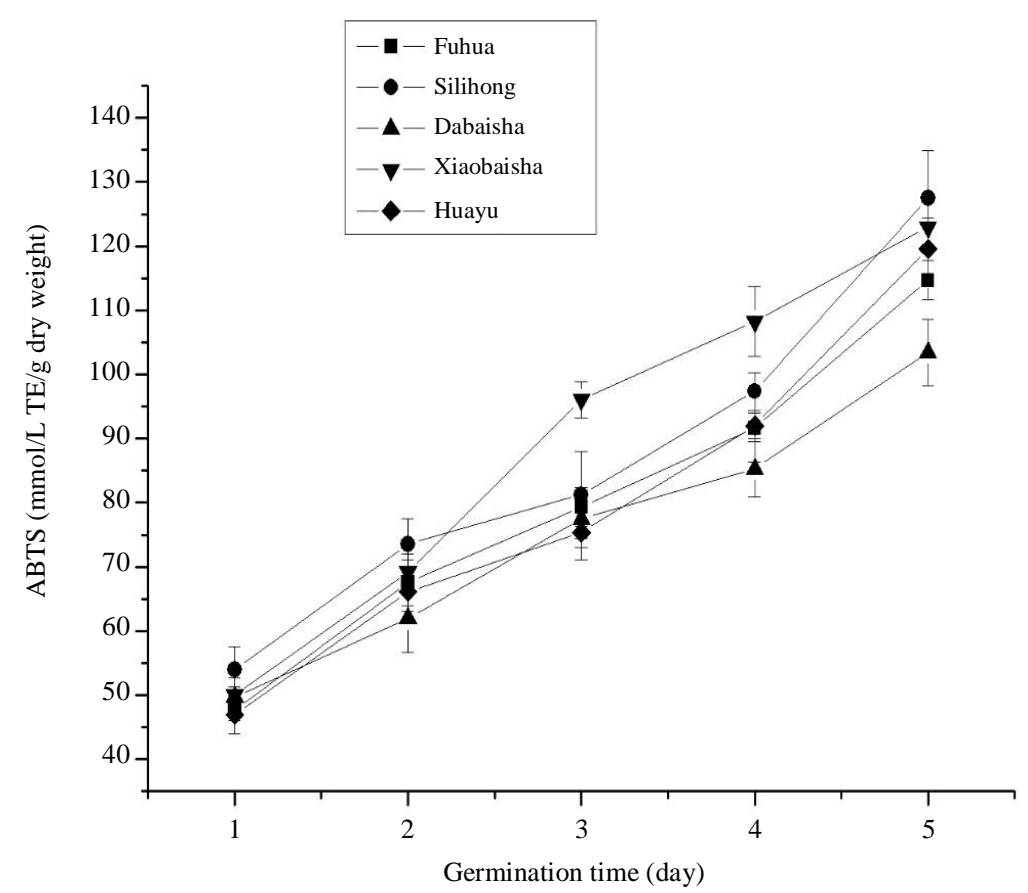

(B) 


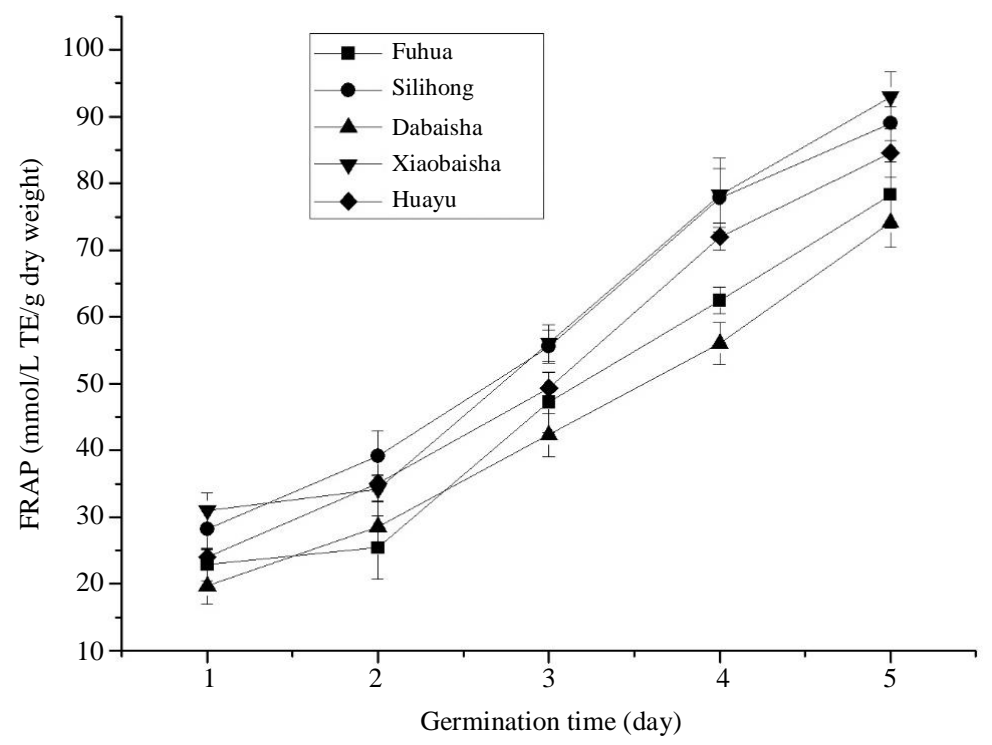

(C)

Fig. 3: Antioxidant capacity of five cultivars of peanut sprout germinated over 1-5 days was expressed as Trolox equivalent. All values are mean $\pm \mathrm{SD}(\mathrm{n}=3)$. A: DPPH assay B: ABTS assay C: FRAP assay

Figure 3 shows the antioxidant activity of the 5 peanut sprouts during germination period. All sprouts showed a similar pattern of all antioxidant activity analysis during seed germination. The antioxidant activity significantly increased to its highest level on the fifth day of germination time. In particular, Xiaobaishan demonstrated the highest level of DPPH [(84.86 \pm 4.02$) \mathrm{mmol} / \mathrm{L} \mathrm{TE} / \mathrm{g}$ dry weight] and FRAP [(92.98 \pm 3.71$) \mathrm{mmol} / \mathrm{L} \mathrm{TE} / \mathrm{g}$ dry weight] antioxidant activity, followed by Silihong, Huayu, Fuhua and Dabaisha. On the other hand, Silihong exhibited the strongest antioxidant activity [(127.49 \pm 7.42$) \mathrm{mmol} / \mathrm{L}$ TE/g dry weight] on ABTS analysis, expressed as approximately two and a half times compared to $(50.37 \pm 3.54) \mathrm{mmol} / \mathrm{L} \mathrm{TE} / \mathrm{g}$ dry weight on the first day of germination. This indicated that antioxidant compounds in Silihong cultivar expressing high reducing ability might be present at this germination stage. Accroding to the results of the total phenolic content and resveratrol content, the results suggested that most of the phenolic compounds and resveratrol determined in the five cultivars could be related to their antioxidant activity. This result was in accordance with the study by Limmongkon et al. (2017) which reported increased scavenging activity for the Tainan 9 cultivar with a shorter germination time. The difference in phenolic content, resveratrol content and antioxidant value between five peanut cultivar sprouts may be strongly influenced by peanut genotype, which produced different complicated structural compositions in each. A wide-ranging biochemical process occurred during seed germination, leading to plant changes during secondary metabolite production.

\section{Antiomicrobial Activity}

Antimicrobial effect of five peanut cultivar sprouts against five foodborne bacteria were tested by the inhibitory zone method and are shown in Table 1. All tested cultivars showed antibacterial effects against five spoilage bacteria. Fuhua showed the highest bactericidal activity against Bacillus subtilis. Silihong showed the strongest inhibitory effects on three Gram-negative bacterial including Escherichia coli, Salmonella enterica and Pseudomonas aeruginosa. Xiaobaisha showed the best antibacterial effect against Staphylococcus aureus subsp. aureus. No effects of extraction reagents were found in any of the strains at the tested concentration.

\section{MICs and MBCs of Five Peanut Cultivar Sprout Extract}

Figure 4 shows the MICs and MBCs of five peanut cultivar sprouts extract against five spoilage bacteria. In general, all peanut cultivars showed higher inhibitory effects against Bacillus subtilis than on other bacteria, with lower MIC and MBC levels. The average MIC and MBC of Silihong against 5 spoilage bacteria were 10.7 and $28.5 \mathrm{mg} / \mathrm{mL}$, indicating a strong antimicrobial effect compared to that of Huayu, with the average MIC and MBC were 15.8 and $50.8 \mathrm{mg} / \mathrm{mL}$, respectively. All sprouts had $<10 \mathrm{mg} / \mathrm{mL}$ MICs on Bacillus subtilis, the lowest MIC was $6.3 \mathrm{mg} / \mathrm{mL}$ obtained from Fuhua. The average MICs of five sprouts against Bacillus subtilis and Staphylococcus aureus subsp. aureus were $7.7 \mathrm{mg} / \mathrm{mL}$ and $11.7 \mathrm{mg} / \mathrm{mL}$, respectively, which were higher than these against Escherichia coli, Salmonella enterica and Pseudomonas aeruginosa, with the average MICs of 13.9 $\mathrm{mg} / \mathrm{mL}, 14.0 \mathrm{mg} / \mathrm{mL}$ and $15.7 \mathrm{mg} / \mathrm{mL}$, respectively. 
Among five peanut cultivars, the inhibitory effects were strongest in Silihong with the average MIC and $\mathrm{MBC}$ values of 10.66 and $28.52 \mathrm{mg} / \mathrm{mL}$, respectively.
These results indicated that all sprouts may exhibit more inhibitory effects on Gram-positive ones, which need to be further investigated in future.

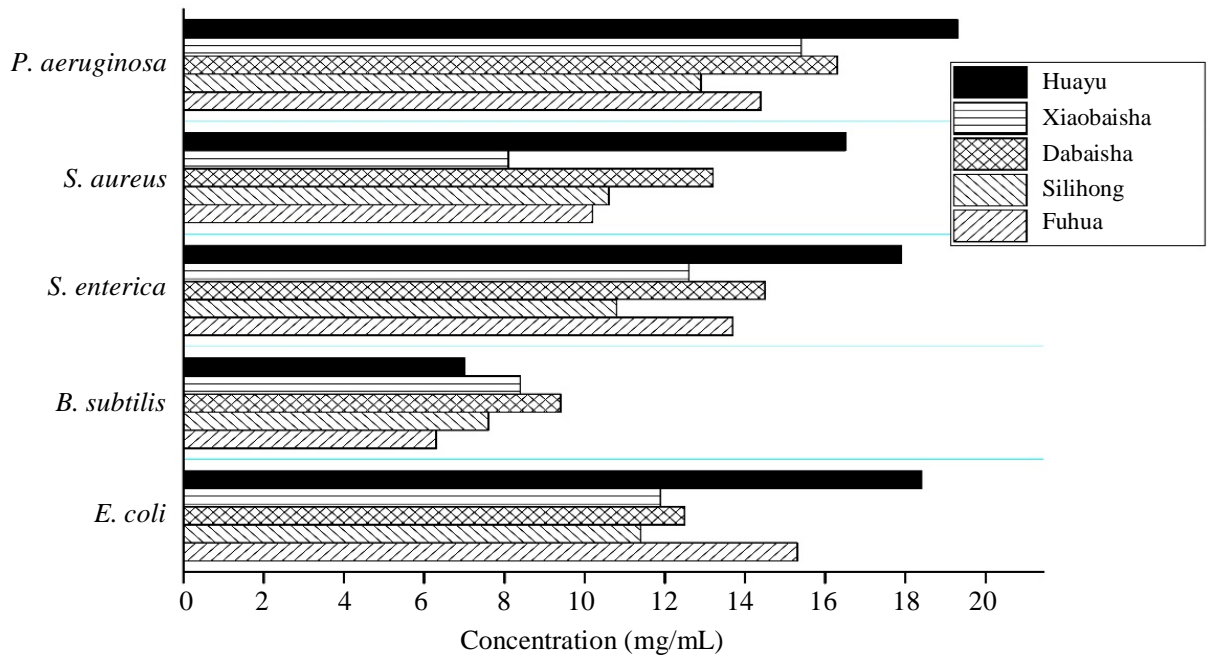

(A)

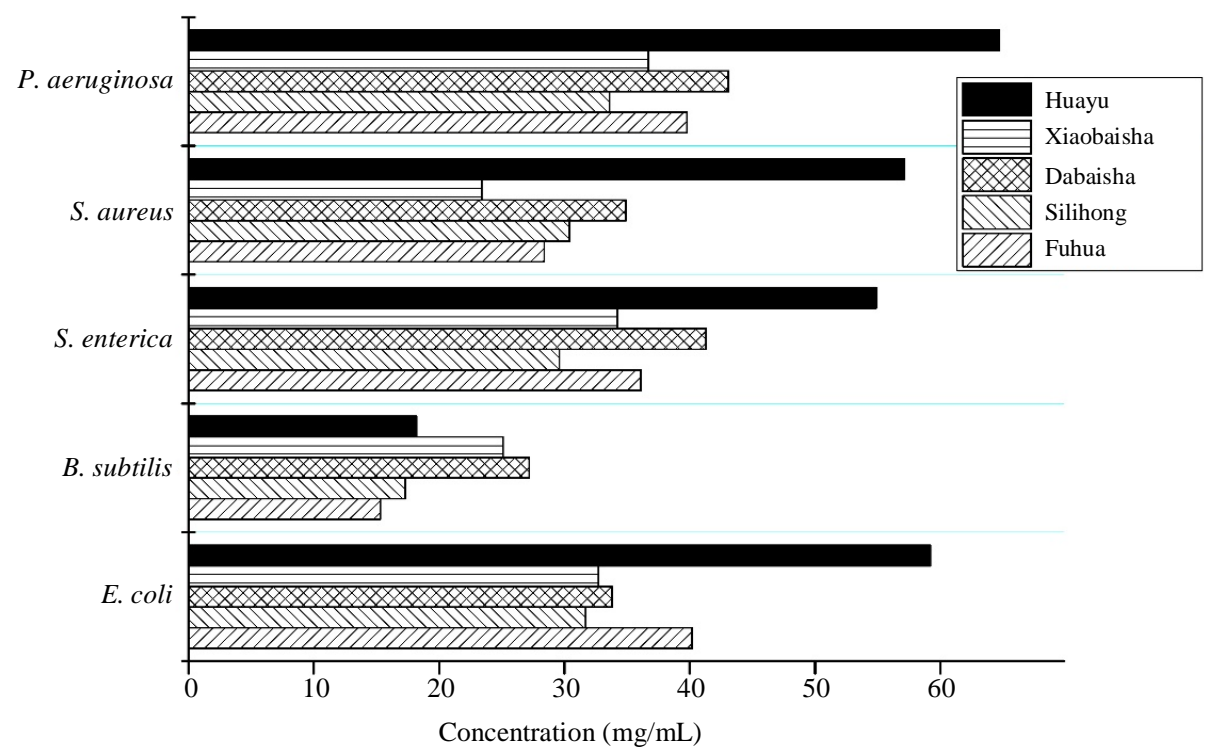

(B)

Fig. 4: Minimum inhibitory concentration (MIC) and minimum bactericidal concentration (MBC) (mg/mL) of five peanut cultivar sprouts against five foodborne bacteria. A: Minimum inhibitory concentration B: Minimum bactericidal concentration. MIC and MBC were determined as the minimum concentration which resulted in optical density at $595 \mathrm{~nm}$, respectively

Table 1: Zones of inhibition $(\mathrm{mm})^{\mathrm{a}}$ around the wells loaded with five cultivar sprout extracts against five foodborne bacteria

\begin{tabular}{|c|c|c|c|c|c|c|}
\hline \multirow[b]{2}{*}{ Spoilage bacteria } & \multirow{2}{*}{$\begin{array}{l}\text { Gram positive/ } \\
\text { negative }\end{array}$} & \multicolumn{5}{|c|}{ Peanut cultivar sprouts } \\
\hline & & Fuhua & Silihong & Dabaisha & Xiaobaisha & Huayu \\
\hline E. coli & $(-)$ & $7.61 \pm 0.12$ & $10.00 \pm 0.41$ & $8.58 \pm 0.34$ & $8.53 \pm 0.22$ & $6.55 \pm 0.35$ \\
\hline B. subtilis & $(+)$ & $10.57 \pm 0.12$ & $8.49 \pm 0.30$ & $7.34 \pm 0.37$ & $7.68 \pm 0.33$ & $9.00 \pm 0.41$ \\
\hline S. enterica & $(-)$ & $7.52 \pm 0.32$ & $9.59 \pm 0.17$ & $7.63 \pm 0.47$ & $8.37 \pm 0.20$ & $6.93 \pm 0.14$ \\
\hline S. subsp. aureus & $(+)$ & $9.11 \pm 0.21$ & $9.04 \pm 0.37$ & $8.25 \pm 0.61$ & $10.06 \pm 0.28$ & $7.12 \pm 0.31$ \\
\hline$P$. aeruginosa & $(-)$ & $8.03 \pm 0.25$ & $10.32 \pm 0.20$ & $7.91 \pm 0.56$ & $8.45 \pm 0.16$ & $6.31 \pm 0.26$ \\
\hline
\end{tabular}

${ }^{\mathrm{a}}$ Diameter $(\mathrm{mm})$ of visual clear zone. 


\section{Conclusion}

The total phenolic compound, resveratrol content and antioxidative activities of five peanut cultivar sprouts were increased significantly with an increase of germination time. The antibacterial activity was evaluated with the inhibitory zone method using the extracts against five foodborne bacterial and all five peanut sprouts showed high antimicrobial activities. The difference in the phenolic content, resveratrol, antioxidant and antimicrobial value between five peanut cultivar sprouts may be strongly influenced by the complicated structural composition of each genotype. Therefore, further studies are required in order to isolate the compounds responsible for its active potential and structural elucidation to find the mechanism of biological activity. Furthermore, in accordance with the bioactive and chemopreventive significance of peanut sprout, it can be developed and consumed as food and medicine in a commercial scale.

\section{Acknowledgment}

This work was supported by the Taishan Industry Leading Talent Project (LJNY201606), Doctor and Postdoctoral Foundation of Shandong University of Technology (4041/414053, 4041/515003).

\section{Author's Contributions}

Qing Liu: Has conceived and designed the experiments, data analysis, manuscript writing and publication.

Shaoqi Li: Has performed the experiments of peanut germination, Total phenolic and resveratrol determination.

Yao Zhang and Huaiyuan Zhang: Have assisted in the experiment.

Haifang Xiao: Has performed the experiments of antioxidant and antimicrobial activity.

Tian Tian: Has donated the peanut sample.

Yuanda Song: Has reviewed and revised the manuscript.

\section{Ethics}

Authors declared no ethical issues that may arise after the publication of this manuscript.

\section{References}

Ahn, Y.J., P. Ganesan and H.S. Kwak, 2012. Comparison of polyphenol content and antiradical scavenging activity in methanolic extract of nanopowdered and powdered peanut sprouts. J. Korean Soc. Applied Biol. Chem., 55: 793-798. DOI: $10.1007 / \mathrm{s} 13765-012-2199-\mathrm{X}$
Akharaiyi, F.C., O.O.O. Odiedi, J.A. Johnson and F.S. Oluwafemi, 2019. Inhibitory effect and preliminary phytochemical screening of some ornamental plants against some bacteria pathogens. Eurasian J. Biosci., 13: 899-908.

EFSA, 2017. ECDC/EFSA/EMA second joint report on the integrated analysis of the consumption of antimicrobial agents and occurrence of antimicrobial resistance in bacteria from humans and food-producing animals. EFSA J., 15: e04872-e04872.

DOI: $10.2903 /$ j.efsa.2017.4872

Ghanim, H., C.L. Sia, K. Korzeniewski, T. Lohano and S. Abuaysheh et al., 2011. A resveratrol and polyphenol preparation suppresses oxidative and inflammatory stress response to a high-fat, highcarbohydrate meal. J. Clin. Endocrinol. Metab., 96: 1409-1414. DOI: 10.1210/jc.2010-1812

Hameed, A., S.A. Hussain, J. Yang, M.U. Ijaz and Q. Liu et al., 2017. Antioxidants potential of the filamentous fungi (Mucor circinelloides). Nutrients, 9: E1101-E1101. DOI: 10.3390/nu9101101

Huang, D., B. Ou and R.L. Prior, 2005. The chemistry behind antioxidant capacity assays. J. Agric. Food Chem., 53: 1841-1856. DOI: 10.1021/jf030723c

Ji, L.L., Y.M. Luo and G.L. Yan, 2008. Studies on the antimicrobial activities of extracts from Eupatorium lindleyanum DC against food spoilage and food-borne pathogens. Food Cont., 19: $995-1001$.

DOI: $10.1016 /$ j.foodcont.2007.10.007

Kim, H.J., K.J. Park and J.H. Lim, 2011. Metabolomic analysis of phenolic compounds in buckwheat (Fagopyrum esculentum M.) sprouts treated with methyl jasmonate. J. Agric. Food Chem., 59: 5707-5713. DOI: 10.1021/jf200396k

Lee, L.S., E.J. Choi, C.H. Kim, J.M. Sung and Y.B. Kim et al., 2016. Contribution of flavonoids to the antioxidant properties of common and Tartary buckwheat. J. Cereal Sci., 68: 181-186.

DOI: 10.1016/j.jcs.2015.07.005

Limmongkon, A., P. Janhom, A. Amthong, M. Kawpanuk and P. Nopprang et al., 2017. Antioxidant activity, total phenolic and resveratrol content in five cultivars of peanut sprouts. Asian Pac. J. Trop. Biomed., 7: 332-338.

DOI: 10.1016/j.apjtb.2017.01.002

Manoharan, A.L., S. Thamburaj, K. Muniyandi, G. Jagadeesan and S. Sathyanarayanan et al., 2019. Antioxidant and antimicrobial investigations of Elaeocarpus tectorius (Lour.) Poir. fruits against urinary tract infection pathogens. Biocatal. Agric. Biotechnol., 20: 101260-101260.

DOI: $10.1016 /$ j.bcab.2019.101260

Nakata, R. and H. Inoue, 2014. Resveratrol and cardiovascular disease. Curr. Nutr. Rep., 3: 163-169. DOI: $10.1007 / \mathrm{s} 13668-014-0084-4$ 
Ożarowski, M., R. Kujawski, P. Mikołajczak, A. Gryszczyńska and A. Pietrowiak et al., 2016. Comparison of antioxidant activities of fractionated extracts from seedlings and herb of Chelidonium majus L. using DPPH, ABTS and FRAP methods. Herba Pol., 62: 22-38. DOI: 10.1515/hepo-2016-0020

Park, S.J., F. Ahmad, A. Philp, K. Baar and T. Williams et al., 2012. Resveratrol ameliorates aging-related metabolic phenotypes by inhibiting cAMP phosphodiesterases. Cell, 148: 421-433. DOI: 10.1016/j.cell.2012.01.017

Prior, R.L., X. Wu and K. Schaich, 2005. Standardized methods for the determination of antioxidant capacity and phenolics in foods and dietary supplements. J. Agric. Food Chem., 53: 4290-4302. DOI: $10.1021 /$ jf0502698

Santos, A.J., G.S.G. de Carvaho, V. Oliveira, N.R.B. Raposo and A.D. da Silva, 2013. Resveratrol and analogues: A review of antioxidant activity and applications to human health. Recent Pat. Food Nutr. Agric., 5: 144-153.

DOI: $10.2174 / 18761429113059990001$

Verraes, C., S.V. Boxstael, E.V. Meervenne, E.V. Coillie and P. Butaye et al., 2013. Antimicrobial resistance in the food chain: a review. Int. J. Environ. Res. Public Health, 10: 2643-2669.

DOI: $10.3390 /$ ijerph10072643
Wang, K.H., Y.H. Lai, J.C. Chang, T.F. Ko and S.L. Shyu et al., 2005. Germination of peanut kernels to enhance resveratrol biosynthesis and prepare sprouts as a functional vegetable. J. Agric. Food Chem., 53: 242-246. DOI: 10.1021/jf048804b

Whitlock, N.C. and S.J. Baek, 2012. The anticancer effects of resveratrol: Modulation of transcription factors. Nutr. Cancer, 64: 493-502. DOI: $10.1080 / 01635581.2012 .667862$

Xiong, Q., Q. Zhang, D. Zhang, Y. Shi and C. Jiang et al., 2014. Preliminary separation and purification of resveratrol from extract of peanut (Arachis hypogaea) sprouts by macroporous adsorption resins. Food Chem., 145: 1-7.

DOI: 10.1016/j.foodchem.2013.07.140

Zhang, Q., Y. Bian, Y. Shi, S. Zheng and X. Gu et al., 2015. An economical and efficient technology for the extraction of resveratrol from peanut (Arachis hypogaea) sprouts by multi-stage countercurrent extraction. Food Chem., 179: 15-25.

DOI: 10.1016/j.foodchem.2015.01.113

Zhang, S.B., 2016. In vitro antithrombotic activities of peanut protein hydrolysates. Food Chem., 202: 1-8. DOI: 10.1016/j.foodchem.2016.01.108 